\title{
PERFIL AUDITIVO DE TRABALHADORES DE UM ENTREPOSTO DE CARNES
}

\section{Workers auditory profile at a meat warehouse}

\author{
Amanda Nogueira Azevedo (1), Luciana Dias Bernardo (2), \\ Suely Cátia Alves Chong Shing ${ }^{(3)}$, Juliana Nunes Santos ${ }^{(4)}$
}

\begin{abstract}
RESUMO
Objetivo: caracterizar o perfil auditivo de trabalhadores de um entreposto de carnes e derivados da região metropolitana de Belo Horizonte. Métodos: trata-se de um estudo transversal descritivo em que foram avaliados 53 trabalhadores desse entreposto, com aplicação de questionário e análise de exames audiométricos. Resultados: dos funcionários avaliados, três $(5,66 \%)$ apresentaram perda auditiva induzida por ruído. Não houve correlação estatisticamente significante entre o tempo de trabalho na empresa e o desenvolvimento de perda auditiva induzida por ruído. Os sintomas auditivos mais encontrados foram dificuldade de entender as palavras $(13,2 \%)$ e intolerância a sons intensos (11,3\%); e os sintomas não auditivos foram dor de cabeça $(16,9 \%)$ e irritabilidade $(11,3 \%)$. Observou-se diferença estatisticamente significante $(p<0,05)$ ao correlacionar os indivíduos com audição normal e alterada quanto à presença de zumbido, queixa de não escutar bem e dificuldade de conversar em ambientes ruidosos. Conclusão: os resultados revelaram que indivíduos com audiometrias sugestivas de perda auditiva induzida por ruído mostraram-se mais vulneráveis ao zumbido, além de apresentarem mais dificuldade de conversar em ambientes ruidosos. No entanto, as demais queixas auditivas e não auditivas foram encontradas em ambos os grupos, independentemente da existência de perda auditiva.
\end{abstract}

DESCRITORES: Perda Auditiva; Ruído; Audiometria; Saúde do Trabalhador; Limiar Auditivo

\section{INTRODUÇÃO}

A audição é um dos sentidos mais importantes para o desenvolvimento psicossocial do indivíduo e tem fundamental importância na integração do homem à sociedade. Dessa forma, tudo o que venha a comprometer esse sentido merece atenção especial ${ }^{1,2}$.

(1) Fonoaudióloga; Especialista em Audiologia pela FEAD Centro de Gestão Empreendedora.

(2) Fonoaudióloga; Especialista em Audiologia pela FEAD Centro de Gestão Empreendedora.

(3) Fonoaudióloga; Audiológica Centro de Prevenção e Diagnóstico Auditivo, Belo Horizonte, MG; Especialista em Audiologia pela FEAD - Centro de Gestão Empreendedora.

(4) Fonoaudióloga; Professora do Curso de Fonoaudiologia da FEAD - Centro de Gestão Empreendedora, Belo Horizonte, MG; Mestre e Doutoranda em Ciências da Saúde da Faculdade de Medicina da Universidade Federal de Minas Gerais e Pesquisadora da Fundação João Pinheiro.

Conflito de interesses: inexistente
Diversos fatores podem levar à perda da audição: doenças metabólicas, vasculares, isquêmicas e iônicas, uso de medicamentos, estresse, predisposição genética, fatores nutricionais, exposição ao ruído, entre outros ${ }^{1}$.

Estudos epidemiológicos sobre perda auditiva no Brasil são escassos e, portanto, não há registros que caracterizem a real situação. Estima-se que $25 \%$ da população trabalhadora exposta seja portadora de perda auditiva induzida por ruído em algum grau ${ }^{3,4}$.

Fazendo uma análise da história, pode-se observar que, após a Revolução Industrial, com o advento da modernização e implantação de máquinas e equipamentos industriais, o ruído nas indústrias aumentou consideravelmente ${ }^{5}$. Com o passar do tempo e com o avanço tecnológico, observouse consequente prejuízo na qualidade de vida dos trabalhadores. Hoje, sabe-se que a exposição ao ruído pode causar sérios danos na qualidade de vida dos indivíduos expostos ${ }^{5,6}$. 
O ruído torna-se fator de risco da perda auditiva se o nível de pressão sonora e o tempo de exposição ultrapassarem certos limites. A Norma Regulamentadora do Ministério do Trabalho, NR 15, estabelece os limites de tolerância para a exposição ao ruído contínuo ou intermitente e para ruído de impacto, vigentes no país. Como regra geral, é tolerada exposição de, no máximo, oito horas diárias, a ruído contínuo ou intermitente, com média ponderada no tempo de 85 dBNA ou uma dose equivalente ${ }^{7}$. A forma como o ruído afeta a audição tem sido alvo de estudo de vários pesquisadores e os resultados desses trabalhos fundamentaram as leis trabalhistas. A Norma Regulamentadora do Ministério do Trabalho, NR 9, estabelece a obrigatoriedade da elaboração e implementação, por parte de todos os empregadores e instituições que admitam trabalhadores como empregados, do Programa de Prevenção de Riscos Ambientais - PPRA, visando à preservação da saúde e da integridade dos trabaIhadores, mediante antecipação, reconhecimento, avaliação e consequente controle da ocorrência de riscos ambientais existentes ou que venham a existir no ambiente de trabalho, tendo em consideração a proteção do meio ambiente e dos recursos naturais ${ }^{7}$. A Portaria 19 da NR 7 tem por objetivos estabelecer diretrizes e parâmetros mínimos para avaliação e acompanhamento da audição do trabalhador por intermédio de realização de exames audiológicos de referência e sequenciais; e fornecer subsídios para a adoção de programas que visem à prevenção da perda auditiva induzida por níveis de pressão sonora elevados (PAINPSE) e a conservação da saúde auditiva dos trabalhadores ${ }^{7}$. Sabe-se que o ruído é inimigo "silencioso" e atinge o homem em vários aspectos. Pessoas aparentemente com boa saúde podem estar sendo vítimas da nocividade do ruído. Por isso, a crescente preocupação com seu monitoramento e controle ${ }^{8}$.

A PAINPSE é a alteração dos limiares auditivos, do tipo sensório-neural, decorrente da exposição ocupacional sistemática a níveis de pressão sonora elevados. Tem como características principais a irreversibilidade e progressão gradual com o tempo de exposição ao risco e, se não houver diminuição ou eliminação desta, o agravamento ${ }^{7}$. Constitui-se, então, um problema de saúde extremamente sério na sociedade ${ }^{9,10}$. A PAINPSE é considerada uma entre as dez principais etiologias de perdas auditivas populacionais, sendo que, de todas as causas de lesão auditiva, é a que apresenta maior possibilidade de prevenção ?

A doença geralmente atinge o seu nível máximo para as frequências de 3,4 e $6 \mathrm{kHz}$ nos primeiros 10 a 15 anos. Após cessada a exposição ao ruído, não haverá progressão da perda ${ }^{11}$.
A exposição ao ruído, acima dos limites de tolerância, tem o poder de lesar desde a membrana timpânica até regiões do Sistema Nervoso Central, e é no órgão de Corti que ocorrem as principais alterações responsáveis pela PAINPSE. As células ciliadas externas, presentes no órgão de Corti, são sensíveis a fortes e prolongadas pressões sonoras, logo a lesão é diretamente proporcional ao tempo em que o indivíduo fica exposto ao ruído. Por esse motivo, intervalos para repouso auditivo em ambientes adequados são fundamentais para tentar recuperar a atividade enzimática das células sensoriais ${ }^{11}$.

Nos efeitos da exposição ao ruído, os danos no aparelho auditivo são bem conhecidos e quantificados, porém as evidências de sua nocividade em outros órgãos e sistemas são crescentes, como alterações físicas e psíquicas. O ruído elevado pode ser causa agravante de doenças no sistema cardiovascular e endócrino por ação do sistema nervoso autônomo ${ }^{11}$. Fatores como sexo, idade e remédios ototóxicos têm sido descritos como agravantes da perda auditiva induzida pelo ruído. As perdas auditivas também podem estar associadas a doenças na família, a otites de repetição, a meningites, ao ruído e podem ser potencializadas pelo uso de solventes e de metais pesados em ocupações pregressas ${ }^{12}$.

Os trabalhadores expostos ao ruído podem apresentar diversas queixas relacionadas à audição, entre as quais se destacam sintomas como hipoacusia, zumbido, plenitude auricular e otalgia, e como sintomas extra-auditivos encontram-se com mais frequência queixas de nervosismo, ansiedade, cefaléia, problemas no estômago e dificuldade para dormir ${ }^{13-15}$.

O ruído encontra-se associado aos acidentes de trabalho, sendo estes os maiores agravos à saúde do trabalhador e, diferentemente do que o nome sugere, não são eventos fortuitos ou acidentais, mas fenômenos socialmente determinados e preveníveis, assim como também são as perdas auditivas induzidas por níveis elevados de pressão sonora. Verifica-se que o ruído é um fator agravante para acidentes de trabalho ${ }^{16,17}$.

As perdas auditivas induzidas por níveis elevados de pressão sonora podem ser evitadas ou estabilizadas por meio do uso de equipamentos de proteção individual (EPI) e da realização de programas de conservação auditiva. Foi observado em estudos que os EPI, quando usados corretamente, provocam mudança nos limiares, sendo fundamentais para evitar a PAINPSE. Destaca-se a importância da implantação de programas de conservação auditiva, a fim de se obter um conjunto de ações individuais e coletivas para controle do ruído e prevenção da perda ${ }^{6,18,19}$. 
Para monitorização da exposição ocupacional ao ruído, a NR7 estabelece que seja feita audiometria admissional, seguida por outro exame após 6 meses e, após estes, periódicos anuais ${ }^{2,7}$.

Monitorar os indivíduos ajuda a identificar evidências precoces do limite da dose para perdas auditivas, levando à possibilidade de prevenção ou detectar precocemente a instalação da perda auditiva. Quando instalada a PAINPSE, existe a possibilidade de reabilitação auditiva com adaptação do aparelho de amplificação sonora individual, minimizando os efeitos da perda na qualidade de vida do indivíduo ${ }^{20}$.

Acredita-se que o conhecimento fonoaudiológico ainda pode contribuir muito para o processo de manutenção da saúde do trabalhador ${ }^{20}$.

Durante o levantamento bibliográfico para este estudo, constatou-se a escassez de trabalhos na área de audiologia relacionados a entreposto de carnes e derivados. Por este, entende-se como o estabelecimento destinado ao recebimento, guarda, conservação, manipulação, acondicionamento e distribuição de carnes frigorificadas das diversas espécies de açougue e outros produtos animais.

O ambiente em questão apresenta níveis de ruído acima do limites de tolerância, merecendo atenção nos estudos em audiologia, pois há indicativo de risco para a saúde auditiva dos trabalhadores, sendo, então, um ambiente onde devam ser aplicadas as legislações do Ministério do Trabalho.

Assim sendo, este trabalho teve por objetivo caracterizar o perfil auditivo de trabalhadores de um entreposto de carnes e derivados da região metropolitana de Belo Horizonte, descrevendo os resultados audiométricos dos trabalhadores, estabelecendo os tipos de perda auditiva e correlacionando os dados obtidos com variáveis de tempo e presença de sintomas auditivos e não auditivos.

\section{MÉTODOS}

Trata-se de um estudo transversal descritivo no qual foram realizadas análises de exames audiométricos e aplicação de questionário em trabalhadores de um entreposto de carnes da região metropolitana de Belo Horizonte.

A amostra do estudo foi composta por todos os 53 trabalhadores da empresa que preencheram os seguintes critérios de inclusão: idade máxima de 50 anos, exposição a níveis de pressão sonora acima de 85 dB NPS na área da desossa e concordância em participar da pesquisa. Foram excluídos aqueles que não concordaram em participar da pesquisa ou que estavam em férias ou licença médica no período da aplicação do questionário ou empregados com idade superior a 50 anos.
O representante da empresa forneceu as medições de ruído feitas anteriormente na área, onde foram encontrados os níveis de 85 dB NPS com pico de 94 dB NPS durante a jornada de trabalho.

Inicialmente, os trabalhadores responderam a um questionário contendo dados de identificação e informações sobre a história de vida e ocupação, antecedentes familiares de perda auditiva, uso de medicamentos, presença de sintomas auditivos e não auditivos, entre outras investigações de possíveis causas de perda de audição. Cada pergunta foi apresentada ao trabalhador oralmente, tendo o examinador a liberdade de parafrasear o conteúdo da questão quando houvesse dificuldade de compreensão por parte do trabalhador entrevistado.

Num segundo momento, foi feita a análise dos resultados dos exames audiométricos pelas pesquisadoras. Os exames foram realizados por uma empresa terceirizada pelo entreposto em abril de 2008.

Para análise dos exames audiométricos, foram consideradas as normas do Comitê Nacional de Ruído e Conservação Auditiva, que caracterizam a Perda Auditiva Induzida por Níveis de Pressão Sonora Elevados (PAINPSE) como:

- Perda auditiva sempre do tipo sensório-neural;

- Perda maior nas frequências agudas, principalmente em 4.000 e $6.000 \mathrm{~Hz}$;

- Perda auditiva quase sempre bilateral;

- Perda na qual, "geralmente", nota-se melhora do limiar na frequência de $8.000 \mathrm{~Hz}$.

Portanto, os resultados audiométricos que atenderam aos critérios descritos acima foram classificados neste estudo como sugestivos de perda auditiva induzida por níveis de pressão sonora elevados ${ }^{7}$.

A interpretação dos resultados do exame audiométrico seguiu os seguintes parâmetros:

- Normais - ou dentro dos padrões de normalidade - casos cujos audiogramas mostrassem limiares auditivos menores ou iguais a $25 \mathrm{~dB}$ NA, em todas as frequências examinadas.

- Alterados - ou sugestivos e não sugestivos de PAINPSE - casos cujos audiogramas mostrassem limiares auditivos maiores que $25 \mathrm{~dB}$ NA, em pelo menos uma das frequências examinadas.

A fim de caracterizar os achados audiométricos, quanto ao tipo de perda auditiva, foram utilizados os critérios estabelecidos pela Portaria 19, de 9 de abril de $1998^{7}$, nas quais as perdas auditivas podem ser divididas em:

- Sugestivas de perda auditiva induzida por níveis de pressão sonora elevados. Nesta categoria se incluíram os audiogramas que atenderam aos seguintes requisitos: presença de limiares auditivos acima de $25 \mathrm{~dB}$ NA nas frequências 
de 3.000 e/ou 4.000 e/ou $6.000 \mathrm{~Hz}$; limiares nas frequências de 3.000 e/ou 4.000 e/ou $6.000 \mathrm{~Hz}$ mais elevados do que nas outras frequências testadas; sendo que as demais frequências testadas poderiam ou não estar comprometidas de acordo com os resultados da via aérea e óssea, uni ou bilateralmente.

- Não sugestivas de Perda auditiva induzida por níveis de pressão sonora elevados. Nesta categoria se incluíram os audiogramas que atenderam aos seguintes requisitos: não se enquadraram na descrição anterior; presença de limiares auditivos menores ou iguais a $25 \mathrm{~dB}$ NA em todas as frequências examinadas.

$\mathrm{Na}$ análise dos audiogramas não foram consideradas diferenças entre dois exames de um mesmo trabalhador, sendo realizada uma análise transversal descritiva.

Este estudo foi aprovado pelo Comitê de Ética em Pesquisa da FEAD, sob protocolo 47, em 22 de fevereiro de 2008. O representante da empresa autorizou a utilização dos dados dos trabalhadores e esses foram esclarecidos quanto aos aspectos voluntários do estudo, seus benefícios e repercussões, através da assinatura de um termo de consentimento livre e esclarecido.

As variáveis analisadas no contexto do estudo foram: resultados dos exames audiométricos, antecedentes de patologias auditivas, tempo de exposição ao ruído, uso de protetor auricular e presença de sintomas auditivos e não auditivos.

Um banco de dados específicos para este trabaIho foi montado no software EPI INFO - 6.04. Para fins de análise descritiva, foi feita a distribuição de frequência das variáveis categóricas envolvidas na avaliação em estudo e análise das medidas de tendência central e de dispersão das variáveis contínuas. Os dados foram previamente conferidos, e os inconsistentes e as informações ignoradas foram tratados adequadamente. Para análise estatística, foram utilizados os testes exatos de Fisher e Anova com valores de $\mathrm{P}<0,05$.

\section{RESULTADOS}

Dos 74 trabalhadores do entreposto, foram investigados 53 , pois $21(28,1 \%)$ foram excluídos por não preencherem os critérios de inclusão. Entre os 53 entrevistados, $36(67,92 \%)$ são do gênero masculino e 17 (32,08\%) do gênero feminino. A média de idade encontrada foi de 30 anos $( \pm 8,1)$, com mínima de 18 anos e máxima de 49 anos.

O tempo médio em que os trabalhadores avaliados estão neste entreposto é de 23,8 meses $( \pm 30,1)$, com tempo mínimo de 3 meses e máximo de 120 meses. Porém, 19 (35,84\%) deles já trabaIharam antes em locais com altos níveis de ruído.

Quanto ao equipamento de proteção individual, $22(41,5 \%)$ trabalhadores relataram fazer o uso de EPI, do tipo plugs e/ou conchas, enquanto $31(58,5 \%)$ relataram não fazer o uso de EPI no ambiente de trabalho. Todos os trabalhadores pesquisados do entreposto estão expostos a elevados níveis de pressão sonora.

Os sintomas auditivos e não auditivos citados pelos trabalhadores do entreposto podem ser visualizados, respectivamente, nas Figuras 2 e 3.

Os resultados das audiometrias dos trabalhadores podem ser visualizados na Figura 4, e as médias dos limiares tonais nas frequências de 250 a 8.000 $\mathrm{Hz}$ encontradas nos trabalhadores na Figura 5.

Na Tabela 1, pode ser visualizado o resultado da análise estatística por meio do Teste Anova, relacionando as variáveis idade e tempo de exposição ao ruído dos trabalhadores com os resultados da audiometria tonal. Os resultados apontaram para a ausência de significância estatística na comparação entre os indivíduos com configurações audiométricas sugestivas ou não de PAINPSE.

As relações das respostas analisadas em anamnese feita com os trabalhadores, comparada aos resultados das audiometrias tonais dos mesmos, estão descritas na Tabela 2.

Foi possível observar que, independentemente da presença de configuração audiométrica sugestiva de PAINPSE, os trabalhadores do entreposto apresentaram características semelhantes no que se refere ao uso de EPI, presença de perda auditiva na família, sintomas não auditivos como tonturas, dores de cabeça, irritabilidade e alterações no sono, além de intolerância a sons intensos.

A análise estatística realizada por meio do Teste Exato de Fisher revelou diferenças estatisticamente significantes $(p<0,05)$ entre os indivíduos com e sem a alteração no exame audiométrico no que concerne a percepção da própria audição, presença de zumbido e dificuldade de conversar em ambientes ruidosos, com sintomas piores nos indivíduos com audiometria alterada. 


\section{ANAMNESE OCUPACIONAL}

Data:

Nome:

Idade:

Sexo: 1- ( ) Masculino 2- ( ) Feminino

1) VOCÊ ESCUTA BEM?

$$
\begin{aligned}
& 1 \text { ( ) Sim } \\
& 2 \text { ( ) Não }
\end{aligned}
$$

2) EXAMES AUDIOMÉTRICOS ANTERIORES:
1 ( ) Sim
2 ( ) Não

3) DADOS OCUPACIONAIS:

3.1 - Empresa onde trabalha:

3.2 - Ramo de atividade:

3.3 - Setor: Tempo:

Nível de Ruído: Setor anterior:

3.4 - Função:

3.5 - Horas diárias de trabalho:

3.6 - Tempo de trabalho na empresa:

3.7 - Exposição a ruído no trabalho (equipamentos barulhentos)?

3.8 - Sente algum mal estar após trabalhar neste ambiente ruidoso?

( ) Sim ( ) Não. Há quanto tempo?

Tempo na empresa:

Tempo total de exposição:

3.9 - Antes deste trabalho atual você trabalhou em algum lugar muito barulhento?

( ) Não ( ) Sim. Por quanto tempo?

3.10 - Pratica alguma atividade barulhenta fora do expediente de trabalho?

( ) Não ( ) Sim. Qual? Com que freqüência?

4) Faz uso de protetor auricular: 1( ) Sim. Desde quando:

$$
\begin{gathered}
1.1 \text { - ( ) Concha } \\
\text { ( ) Plug } \\
\text { ( ) Outros }
\end{gathered}
$$

$$
2 \text { ( ) Não }
$$

Se SIM, responda:

Atualmente, com qual freqüência? ( ) de vez em quando

$$
\text { ( ) quase sempre que exposto ao barulho }
$$

( ) sempre que exposto ao barulho

5) ANTECEDENTES PESSOAIS:

5.1 - Dor de Ouvido: 1 ( ) Sim ( ) OD ( ) OE ( ) Bilateral

$$
2 \text { ( ) Não }
$$

5.2 - Secreção no ouvido: 1 ( ) Sim ( ) OD ( ) OE ( ) Bilateral

2 ( ) Não

3 ( ) Não sabe

5.3 - Toma algum medicamento? Sim ( ) Qual:

Não ( ) 
5.4 - Você percebeu alguma mudança na sua audição depois de alguma doença séria?

( ) Não ( ) Sim. Qual doença?

5.5 - Você notou alguma diferença na sua audição depois que tomou algum remédio?

( ) Não ( ) Sim ( ) Melhorou ( ) Piorou

5.6 - Cirurgia de orelha:

$1-($ ) Não

$2-($ ) Sim, bilateral

$3-($ ) Sim, OD

$4-($ ) Sim, OE

5 - ( ) Sim, não sabe referir o lado

6) SOFREU TRAUMA CRANIANO:

1( ) Sim 2( ) Não 3( ) Não sabe

7) SOFREU TRAUMA ACÚSTICO:

1( ) Sim 2( ) Não 3( ) Não sabe

8) ALGUMA PESSOA NA FAMÍLIA TEM PROBLEMA AUDITIVO:

1( ) Sim 2( ) Não 3 ( ) Não sabe. Qual grau de parentesco?

9) TONTURA:

1 ( ) Sim 2 ( ) Não Classifique seu sintoma: 0- baixo 1- médio 2- alto

10) INTOLERÂNCIA A SONS INTENSOS

1( ) Sim 2( ) Não Classifique seu sintoma: 0- baixo 1- médio 2- alto

11) ZUMBIDO:

1 ( ) Sim 2( ) Não Classifique seu sintoma: 0- baixo 1- médio 2- alto

( ) orelha direita ( ) orelha esquerda ( )ambas

Com que freqüência?

Desde quando?

12) DOR DE CABEÇA:

1 ( ) Sim 2( ) Não Classifique seu sintoma: 0- baixo 1- médio 2- alto

13) IRRITABILIDADE:

1 ( ) Sim 2( ) Não Classifique seu sintoma: 0- baixo 1- médio 2-alto

14) DIFICULDADE DE ENTENDER AS PALAVRAS:

1 ( ) $\operatorname{Sim} 2$ ( ) Não Classifique seu sintoma: 0- baixo 1- médio 2- alto

15) É DESATENTO?

1 ( ) Sim 2 ( ) Não Classifique seu sintoma: 0- baixo 1- médio 2- alto

16) DIFICULDADE DE CONVERSAR EM AMBIENTES RUIDOSOS:

1 ( ) Sim 2( ) Não Classifique seu sintoma: 0- baixo 1- médio 2- alto

17) ALTERAÇÕES NO SONO

1( ) Sim 2( ) Não Classifique seu sintoma: 0- baixo 1- médio 2- alto

Figura 1 - Questionário adaptado do padrão de: Morata TC e Fernandes M (2002)

Rev. CEFAC, São Paulo 


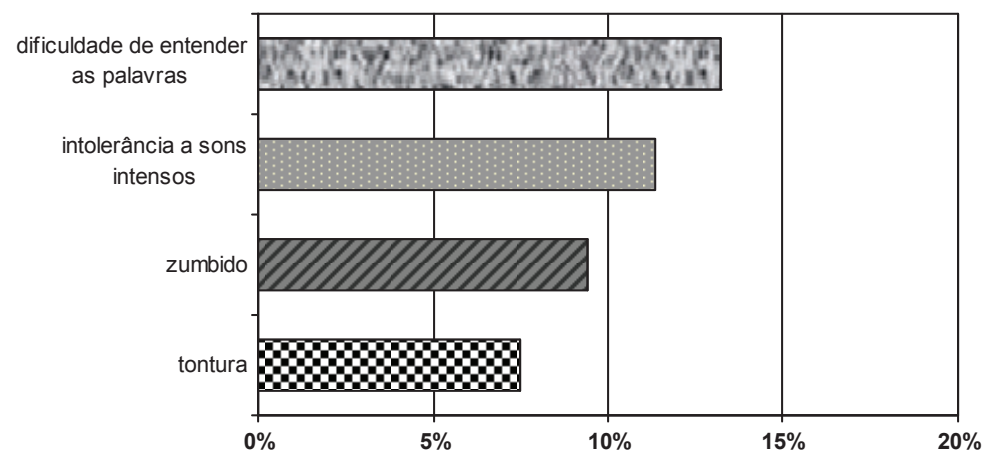

Figura 2 - Sintomas auditivos relatados pelos 53 trabalhadores de um entreposto de carnes e derivados da região metropolitana de Belo Horizonte

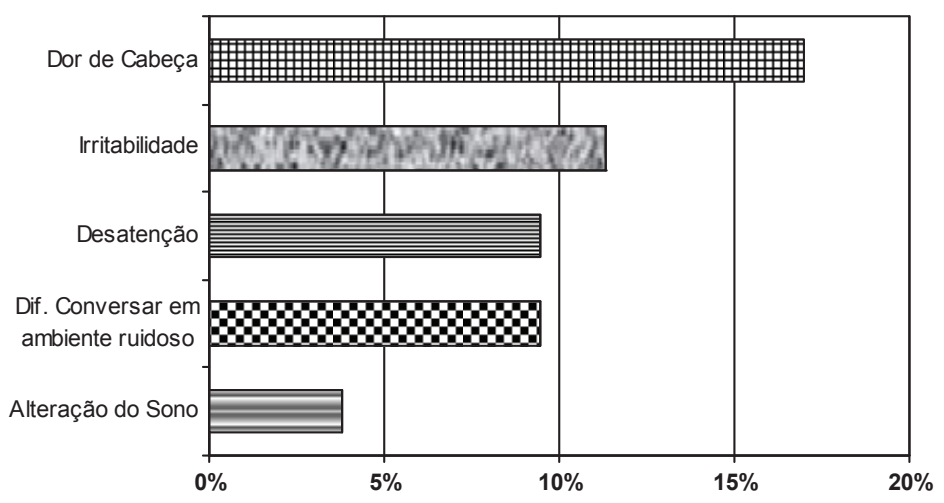

Figura 3 - Sintomas extra-auditivos relatados pelos 53 trabalhadores de um entreposto de carnes e derivados da região metropolitana de Belo Horizonte

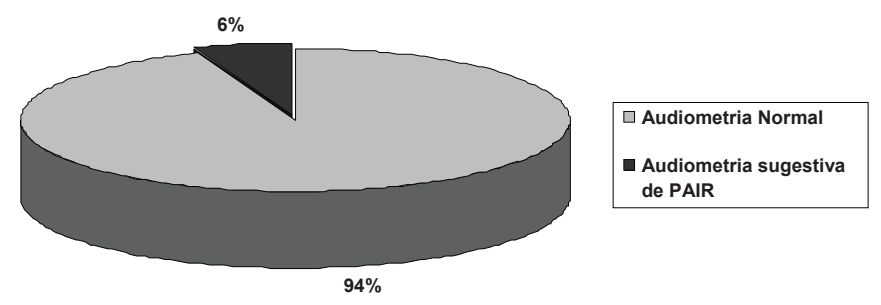

Figura 4 - Resultado das audiometrias dos trabalhadores do entreposto de carnes e derivados da região metropolitana de Belo Horizonte

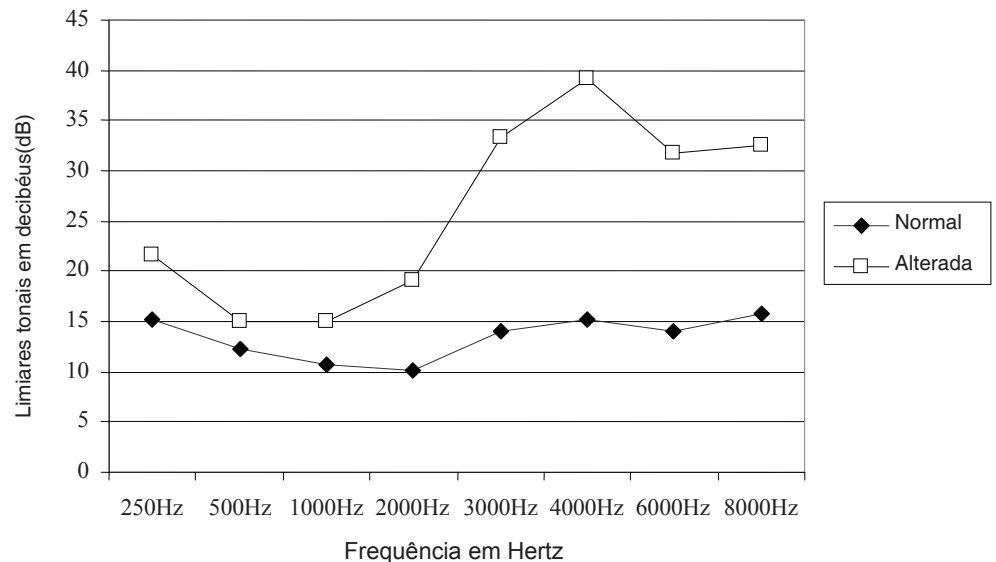

Figura 5 - Médias dos limiares tonais dos exames audiométricos dos trabalhadores do entreposto de carnes e derivados da região metropolitana de Belo Horizonte, randomizados em dois grupos 
Tabela 1 - Associação das variáveis dependentes com a idade e tempo total de exposição ao ruído em relação às audiometrias normais e sugestivas de PAIR

\begin{tabular}{|c|c|c|c|c|c|c|c|}
\hline \multirow{2}{*}{ Resultados } & \multicolumn{2}{|c|}{ Áudiometria normal } & \multicolumn{2}{|c|}{$\begin{array}{c}\text { Áudiometria sugestivo } \\
\text { de PAIR }\end{array}$} & \multirow{2}{*}{ Diferença } & \multirow{2}{*}{ Anova } & \multirow{2}{*}{$\mathbf{P}$} \\
\hline & Média & $\begin{array}{l}\text { Desvio- } \\
\text { padrão }\end{array}$ & Média & $\begin{array}{l}\text { Desvio- } \\
\text { padrão }\end{array}$ & & & \\
\hline Idade (anos) & 29,6 & 8,0 & 35,3 & 10,6 & 5,6 & 1,35 & 0,24 \\
\hline $\begin{array}{l}\text { Tempo de exposição } \\
\text { (meses) }\end{array}$ & 24,5 & 30,8 & 12,0 & 5,1 & 12,5 & 0,49 & 0,48 \\
\hline
\end{tabular}

*Teste de Anova . Valor $p<0,05$

Tabela 2 - Distribuição dos sintomas auditivos e não auditivos entre os 53 trabalhadores do entreposto, randomizados em dois grupos segundo alteração no exame audiométrico

\begin{tabular}{|c|c|c|c|c|c|c|c|}
\hline & & Normal & $\%$ & Alterada & $\%$ & Teste & $\mathbf{P}$ \\
\hline \multirow{2}{*}{ Escuta Bem } & $\operatorname{Sim}$ & 50 & $100 \%$ & 2 & $66,6 \%$ & \multirow{2}{*}{3,75} & \multirow{2}{*}{$0,05^{\prime}$} \\
\hline & Não & 0 & $0 \%$ & 1 & $33,3 \%$ & & \\
\hline \multirow{2}{*}{ Uso do EPI } & $\operatorname{Sim}$ & 20 & $40 \%$ & 2 & $66,6 \%$ & \multirow{2}{*}{0,83} & \multirow{2}{*}{0,37} \\
\hline & Não & 30 & $60 \%$ & 1 & $33,3 \%$ & & \\
\hline \multirow{2}{*}{$\begin{array}{l}\text { Perdas Auditivas } \\
\text { na Família }\end{array}$} & Sim & 1 & $2 \%$ & 0 & $0 \%$ & \multirow{2}{*}{0,04} & \multirow{2}{*}{0,94} \\
\hline & Não & 49 & $98 \%$ & 3 & $100 \%$ & & \\
\hline \multirow{2}{*}{ Tonturas } & $\operatorname{Sim}$ & 4 & $8 \%$ & 0 & $0 \%$ & \multirow{2}{*}{0,38} & \multirow{2}{*}{0,53} \\
\hline & Não & 46 & $92 \%$ & 3 & $100 \%$ & & \\
\hline \multirow{2}{*}{$\begin{array}{l}\text { Intolerância a Sons } \\
\text { Intensos }\end{array}$} & Sim & 6 & $12 \%$ & 0 & $0 \%$ & \multirow{2}{*}{0,09} & \multirow{2}{*}{0,76} \\
\hline & Não & 44 & $88 \%$ & 3 & $100 \%$ & & \\
\hline \multirow{2}{*}{ Zumbido } & $\operatorname{Sim}$ & 3 & $6 \%$ & 2 & $66,6 \%$ & \multirow{2}{*}{6,12} & \multirow{2}{*}{$0,02^{\prime}$} \\
\hline & Não & 47 & $94 \%$ & 1 & $33,3 \%$ & & \\
\hline \multirow{2}{*}{ Dor de Cabeça } & Sim & 8 & $16 \%$ & 1 & $33,3 \%$ & \multirow{2}{*}{0,59} & \multirow{2}{*}{0,43} \\
\hline & Não & 42 & $84 \%$ & 2 & $66,6 \%$ & & \\
\hline \multirow{2}{*}{ Irritabilidade } & $\operatorname{Sim}$ & 5 & $10 \%$ & 1 & $33,3 \%$ & \multirow{2}{*}{0,09} & \multirow{2}{*}{0,76} \\
\hline & Não & 45 & $90 \%$ & 2 & $66,6 \%$ & & \\
\hline \multirow{2}{*}{$\begin{array}{l}\text { Dificuldade de } \\
\text { Entender Palavras }\end{array}$} & Sim & 6 & $12 \%$ & 1 & $33,3 \%$ & \multirow{2}{*}{1,10} & \multirow{2}{*}{0,29} \\
\hline & Não & 44 & $88 \%$ & 2 & $66,6 \%$ & & \\
\hline \multirow{2}{*}{ Desatenção } & Sim & 5 & $10 \%$ & 0 & $0 \%$ & \multirow{2}{*}{0,19} & \multirow{2}{*}{0,65} \\
\hline & Não & 45 & $90 \%$ & 3 & $100 \%$ & & \\
\hline \multirow{2}{*}{$\begin{array}{l}\text { Dificuldade de } \\
\text { Conversar em } \\
\text { Ambiente Ruidoso }\end{array}$} & Sim & 3 & $6 \%$ & 2 & $66,6 \%$ & \multirow[b]{2}{*}{6,42} & \multirow[b]{2}{*}{$0,02^{*}$} \\
\hline & Não & 47 & $94 \%$ & 1 & $33,3 \%$ & & \\
\hline Alteracõe & Sim & 2 & $4 \%$ & 0 & $0 \%$ & 012 & ه \\
\hline Alteraçoes no sono & Não & 48 & $96 \%$ & 3 & $100 \%$ & 0,12 & 0,88 \\
\hline
\end{tabular}

Teste de Fisher. Valor $\mathrm{p}<0,05$.

\section{DISCUSSÃO}

Dos 53 trabalhadores avaliados, 33\% são do gênero feminino, diferentemente dos estudos feitos com trabalhadores expostos ao ruído em diversas áreas da indústria que avaliaram apenas trabalhadores do gênero masculino $1,2,6,14,17,19,21$. Na literatura, encontram-se evidências de que o gênero pode aumentar a susceptibilidade do indivíduo ao ruído e agravar a PAINPSE ${ }^{1,11}$. No estudo realizado com trabalhadores de um distrito industrial, observou-se que $84,1 \%$ deles eram do sexo masculino, constatando nestes a prevalência de perda auditiva sensório-neural $(11 \%){ }^{9}$, corroborando achados de outros estudos ${ }^{14,22}$. Há maior ocorrência de PAINPSE no gênero masculino devido, provavelmente, ao maior percentual de homens expostos a ruídos ocupacionais ${ }^{9,10,13,22}$. O presente estudo não diverge do que é comumente encontrado, mostrando que $67,92 \%$ dos trabalhadores são do sexo masculino. 
Neste estudo, a idade máxima encontrada nos trabalhadores foi de 49 anos. A literatura apresenta estudos de prevalência da PAINPSE, em diferentes locais de trabalho, com amostras incluindo idades mais elevadas ${ }^{9,13,16,17,23}$. Estudos mostram que acima de 50 anos de idade os indivíduos expostos ao ruído têm mais riscos de desenvolverem perda auditiva sensório-neural ${ }^{9,13,24}$. A exclusão de trabaIhadores com mais de 50 anos descartou a possibilidade das perdas auditivas serem confundidas com uma presbiacusia, que é um fenômeno biológico de alta prevalência, e torna-se socialmente incômodo a partir dos 50 anos ${ }^{25}$.

O tempo de trabalho dos avaliados no entreposto foi de 23,8 meses. De acordo com o Comitê de Ruído e Conservação da Audição, esse tempo não é suficiente para desencadear PAINPSE, que começa a se desenvolver no período de 5 a 7 anos de exposição contínua a níveis elevados de ruído e atinge seu nível máximo nos primeiros 10 a 15 anos ${ }^{11,13}$, corroborando outras literaturas ${ }^{1,2,4,6,14}$.

Um estudo realizado em distrito industrial, assim como este presente estudo, não foram excluídos de sua amostra funcionários com baixo tempo de exposição, porém mostrou, em sua análise, numa pesquisa de avaliação de dados de exames audiométricos, que acima de 10 anos de exposição ao ruído a porcentagem de exames alterados sugestivos de PAINPSE dobrou, passando de $14,3 \%$ nos primeiros 10 anos a $30,7 \%$ na segunda década de exposição ao ruído ${ }^{9}$, justificando, assim, o baixo índice de PAINPSE encontradas neste estudo.

Percebe-se, então, que é necessária maior atenção em trabalhadores com tempo de exposição superior a cinco anos. No entanto, o acompanhamento de funcionários, independentemente do tempo de exposição, é imprescindível para prevenir o desencadeamento da PAINPSE e promover melhora da qualidade de vida.

Neste estudo, 31 trabalhadores $(58,5 \%)$ declararam não fazer uso do EPI. Dados semelhantes foram encontrados em trabalhadores de uma indústria de bebidas, onde somente $31 \%$ faziam uso de EPI ${ }^{2}$. Entretanto, o uso do EPI é proteção que pode mostrar melhora significativa na frequência de $4 \mathrm{kHz}$, se utilizado corretamente ${ }^{8}$. Um estudo em uma indústria de cerâmica mostra que o uso do EPI em bom estado de conservação inibe a mudança temporária no limiar pós-jornada de trabalho - ao contrário do protetor em mau estado de conservação, em que foi possível observar aumento dos limiares nas frequências de $500 \mathrm{~Hz}, 1.000 \mathrm{~Hz}$ e $4.000 \mathrm{~Hz}{ }^{18}$. No entanto, mesmo com o uso do EPI, pode-se encontrar PAINPSE caso estes não estejam sendo usados corretamente, pois ele provoca maior atenuação nos indivíduos que são treinados para seu uso correto ${ }^{14,17}$. No entreposto estudado, observou-se baixo nível de adesão à proteção auditiva, o que aponta a necessidade de implantação de programas educativos e treinamento para o uso dos protetores, que são fundamentais para a prevenção da PAINPSE ${ }^{18}$.

Nos funcionários analisados, os sintomas auditivos encontrados em maior prevalência foram: dificuldade de entender as palavras, intolerância a sons intensos, zumbido e tontura (Figura 2). Esses dados discordam dos estudos ${ }^{10,12,14-16,23}$, nos quais 0 zumbido apresentou-se como a queixa auditiva com maior freqüência. Tal fato, possivelmente, justificase pelo perfil dos trabalhadores investigados. Além disso, o ruído é um dos fatores etiológicos do zumbido, o qual pode ser decorrente de outros fatores.

Estudo realizado com funcionários de indústria na cidade de Marília também encontrou os sintomas de zumbido $(68,7 \%)$, intolerância a sons intensos $(56,2 \%)$ e dificuldades de compreensão da fala $(43,7 \%)$. No entanto, observou-se uma ocorrência mais alta nesses trabalhadores, o que pode ser justificado pelo tempo de exposição ao ruído ocupacional - que foi num período mínimo de sete anos - como critério de inclusão da pesquisa ${ }^{2}$. Os metalúrgicos do município de Goiânia, referiram o zumbido (13\%), tontura, dificuldade de compreensão da fala (12\%) e hipoacusia (7\%) como frequentes ${ }^{11}$ - sintomas semelhantes aos encontrados nos trabalhadores do entreposto. No estudo em trabaIhadores de uma indústria têxtil ${ }^{13}$, o zumbido foi encontrado em $9,6 \%$ dos sujeitos, e a tontura em $3,8 \%$, e maior ocorrência na dificuldade em entender as palavras $(25 \%)$ - dados semelhantes aos encontrados no presente estudo. Os dados citados mostram que a ocorrência do zumbido pode acontecer com maior ou menor intensidade variando de acordo com o tempo de exposição, o tipo de ruído no local de trabalho, além de características individuais do sujeito.

Percebe-se, portanto, que as queixas auditivas estão presentes em indivíduos expostos ao ruído em diferentes contextos ocupacionais, podendo variar de acordo com o local de trabalho e grau de exposição. Neste sentido, a adoção de medidas preventivas à saúde dos trabalhadores deve ser prioridade nas empresas.

A partir da análise dos resultados do presente estudo é possível perceber que os trabalhadores do entreposto de carnes, por sua exposição, tornamse vulneráveis às queixas de alterações auditivas. Ressalta-se que foram encontradas, juntamente com o zumbido, outras queixas de menor ocorrência nos indivíduos expostos a níveis elevados de ruído, independente da presença da perda auditiva. 
No estudo com a população exposta a níveis de pressão sonora elevados e vibração, pode-se observar, entre as queixas mais comuns, a presença de nervosismo, ansiedade e cefaleia ${ }^{15}$.

Percebe-se, então, que trabalhadores de diversas áreas da indústria, mesmo não apresentando alteração auditiva, têm se queixado de sintomas não auditivos, o que mostra uma estreita relação entre exposição a níveis elevados de pressão sonora e alteração da qualidade de vida dos trabalhadores.

A análise dos exames audiométricos dos trabalhadores revelou que 5,6\% apresentam alterações sugestivas de PAINPSE (Figura 4), o que não se relacionou de maneira estatisticamente, segundo o Teste Anova, com o tempo de exposição (Tabela 1).

Dos três $(5,66 \%)$ exames alterados encontrados neste estudo, dois $(66,6 \%)$ deles dizem respeito a pessoas que fazem uso do equipamento de proteção auditivo e outro $(33,3 \%)$ é relativo a quem não faz uso de proteção, porém dois $(66,6 \%)$ deles se referenciavam a indivíduos que relataram ter trabalhado em locais com altos níveis de ruído, antes de assumirem cargos no entreposto. Quanto aos funcionários que usavam EPI e apresentaram perda sugestiva de PAINPSE, esse dado pode ser justificado pela falta de treinamento para uso correto e cuidados com os EPI, assim como no estudo retrospectivo, no qual $100 \%$ dos trabalhadores da pesquisa faziam o uso do EPI por norma obrigatória na empresa, porém, $43 \%$ apresentaram alterações nas faixas de 3.000 a $6.000 \mathrm{~Hz}$, o que foi justificado pelo ineficaz treinamento dos funcionários ${ }^{14}$.

Percebe-se, com os dados encontrados, que, mesmo com o uso obrigatório do EPI, é necessário um trabalho de conscientização sobre os danos irreversíveis que uma perda auditiva induzida por ruído pode causar. $E$ isso requer que a empresa ofereça treinamento aos seus trabalhadores a respeito do uso correto de seus equipamentos de proteção.

A análise dos limiares tonais dos trabalhadores (Figura 5) mostrou que as frequências mais afetadas nos indivíduos com audiometrias sugestivas de PAINPSE foram de 3, 4, 6 e $8 \mathrm{kHz}$. As alterações nessas frequências são encontradas na PAINPSE, segundo o critério da Portaria $19{ }^{8}$.

Encontrou-se concordância entre os dados obtidos no presente estudo e outras pesquisas no que se refere à frequência mais acometida nos casos sugestivos de PAINPSE, que foi a de $4000 \mathrm{~Hz}{ }^{1,2,11,14,15}$, podendo comprovar os critérios estabelecidos pela Portaria $19^{8}$.

Os resultados deste trabalho discordam do estudo em empresa frigorífica volátil, no qual a frequência mais acometida foi a de $6000 \mathrm{~Hz}{ }^{22}$, e do estudo em trabalhadores de um abatedouro de aves que revela uma piora no limiar de $8.000 \mathrm{~Hz}-\mathrm{e}$ isso não estaria relacionado com as características iniciais de PAINPSE ${ }^{6}$.

Percebe-se, então, que, com evolução do tempo de exposição ao ruído, a tendência é que estejam comprometidas as frequências entre 3.000 e 8.000 $\mathrm{Hz}$. Entalhes nas frequências altas podem ser um alerta para intervenção precoce na saúde auditiva desses trabalhadores, visto que aquelas são as frequências de acometimento primário numa perda por exposição ao ruído.

No presente estudo, encontrou-se associação estatisticamente significante, pelo Teste exato de Fisher, entre a queixa de audição e os resultados audiométricos (Tabela 2). O mesmo ocorreu em relação ao zumbido e à dificuldade de conversar em ambientes ruidosos - sintomas que se mostraram presentes em indivíduos com perda auditiva induzida por ruído. Em trabalhadores de setores com altos níveis de ruído, 83,3\% dos indivíduos relataram não escutar bem ${ }^{23}$. Como já citado anteriormente, vários são os estudos em que o zumbido aparece como queixa principal, quando se trata de exposição ao ruído ${ }^{23}$. Esses dados foram semeIhantes aos encontrados no presente estudo, já que o zumbido também esteve presente em indivíduos sem perda, mostrando que esse sintoma pode ser um sinal de alerta que antecede a perda.

Observou-se, com os achados deste estudo, que, mesmo com a baixa ocorrência de perdas auditivas induzidas por ruído, muitas foram as queixas auditivas e não auditivas relatadas pelos trabaIhadores do entreposto. Tais achados podem estar relacionados a uma futura PAINPSE, o que mostra que, desde já, devem ser tomadas medidas preventivas para proteção da saúde auditiva dos trabalhadores. É necessária a implantação de um programa de conservação auditiva, o qual se justifica pelos achados desta pesquisa. Além disso, o PCA possibilita preservar a audição e minimizar qualquer tipo de sintoma que venha a se relacionar com a exposição ao ruído, melhorando, dessa forma, a qualidade de vida dos trabalhadores.

\section{CONCLUSÃO}

Neste estudo, caracterizou-se o perfil auditivo dos trabalhadores de um entreposto de carnes e derivados, descrevendo os resultados audiométricos, correlacionando-os com as variáveis de tempo e sintomas auditivos e não auditivos apresentados pelos trabalhadores.

Os trabalhadores do entreposto de carnes e derivados apresentaram, em sua maioria $(94,3 \%)$, resultados audiométricos dentro do padrão de normalidade em todas as frequências. 
Entre os indivíduos com audiometrias alteradas, todos apresentaram configurações audiométricas sugestivas de perda auditiva induzida por ruído com entalhe entre 3 e $6 \mathrm{kHz}$.

Não houve correlação estatisticamente significante, por meio do teste de Anova, entre o tempo de trabalho na empresa e o desenvolvimento de perda auditiva induzida por ruído.

Os resultados revelaram que indivíduos com audiometrias sugestivas de PAINPSE mostraramse mais vulneráveis ao zumbido, além de apresentarem mais dificuldade de conversar em ambientes ruidosos. No entanto, as demais queixas auditivas e não auditivas foram encontradas em ambos os grupos, independentemente da existência de perda auditiva.

Acredita-se que o ruído deva ser encarado com mais seriedade pelos empregadores e empregados, e que se deve investir em treinamentos sobre o correto uso dos equipamentos de proteção e o cuidado necessário com eles, além de se trabalhar a conscientização da alteração que o ruído pode trazer para a saúde auditiva quando se está exposto a ele por muito tempo. O investimento em implantação de programas de conservação auditiva possibilita a minimização dos sintomas auditivos e não auditivos, assim como a ocorrência da PAINPSE, promovendo melhor qualidade de vida ao trabalhador.

\begin{abstract}
Purpose: to characterize the auditory profile of workers at a Meat and Derivative Warehouse of the metropolitan region of Belo Horizonte. Methods: it is a transversal study where 53 workers of this warehouse had been evaluated, with application of questionnaire and analysis of audiometric examinations. Results: from the evaluated employees, $5.66 \%$ showed induced auditory loss for noise. The exposition time did not relate with induced auditory loss for noise. The most common auditory symptoms had been difficulty to understand words (13.2\%) and difficulty to bear the intense sounds $(11.3 \%)$ and the not auditory symptoms had been headache $(16.9 \%)$ and irritability $(11.3 \%)$. Significant difference was observed $(p<0.05)$ when correlating the individuals with normal and modified hearing with the presence of humming, complaint about listening abilities and difficulty to talk in noisy environments. Conclusion: the results reveled that individuals with audiometries suggesting induced auditory loss for noise were more vulnerable to humming, also showing more difficulty to talk in noisy environments. However, the others auditory and non-auditory complaints were raised in both groups, regardless of the existence of auditory loss.
\end{abstract}

KEYWORDS: Hearing Loss; Noise; Audiometry; Occupational Health; Auditory Threshold

\section{REFERÊNCIAS}

1. Siviero AB, Fernandes MJ, Lima JAC, Santoni CB; Bernardi APA. Prevalência de perda auditiva em motoristas de ônibus do transporte coletivo da cidade de Maringá-PR. Rev CEFAC. 2005; 7(3):376-81.

2. Guida HL. Efeitos psicossociais da perda auditiva induzida pelo ruído em ex-funcionários da indústria. Acta ORL. 2007; 25(1):78-83.

3. Brasil. Ministério da Saúde. Secretaria de Atenção à Saúde. Departamento de Ações Programáticas Estratégicas. Perda auditiva induzida por ruído (PAIR). Brasília: Editora do Ministério da Saúde; 2006. p. 1-40.

4. Guerra MR, Lourenço PMC, Teixeira MTB, Alves MJM. Prevalência de perda auditiva induzida por ruído em empresa metalúrgica. Rev Saúde Pública. 2005; 39(2):238-44.
5. Rouquayrol MZ, Almeida Filho N. Epidemiologia e saúde. 6. ed. Rio de Janeiro: Ed. Médica e Científica Ltda; 2003. p. 37-415.

6. Basso VB, Campos AL, Thiessen J. Estudo da alteração temporária do limiar auditivo em trabalhadores expostos ao ruído. Rev CEFAC. 2003; 5(2):187-93.

7. Equipe atlas. Segurança e medicina do trabalho. 54. ed. São Paulo: Atlas; 2004.

8. Rodrigues MAG, Dezan AA, Marchiori LLM. Eficácia da escolha do protetor auditivo pequeno, médio e grande em programa de conservação auditiva. Rev CEFAC. 2006; 8(4):543-7. dx.doi. org/10.1590/S1516-18462006000400016

9. Teles RM, Medeiros MPH. Perfil audiométrico de trabalhadores do distrito industrial de Maracanaú CE. Rev Soc Bras Fonoaudiol. 2007; 12(3):233-9.

10. Dias A, Cordeiro R, Corrente JE, Gonçalves CGO. Associação entre perda auditiva induzida 
pelo ruído e zumbidos. Cad Saúde Pública. 2006; 22(1):63-8.

11. Araújo SA. Perda auditiva induzida pelo ruído em trabalhadores de metalúrgica. Rev Bras Otorrinolaringol. 2002; 68(1):47-52.

12. Corrêa Filho HR, Costa LS, Hoehne EL, Pérez MAG, Nascimento LCR, Moura EC. Perda auditiva induzida por ruído e hipertensão em condutores de ônibus. Rev Saúde Pública. 2002; 36(6):693-701.

13. Caldart AU, Adriano CF, Terruel I, Martins RF, Caldart AU, Mocellin M. Prevalência da perda auditiva induzida pelo ruído em trabalhadores de indústria têxtil. Arq Int Otorrinolaringol. 2006; 10(3):192-6.

14. Amaral M, Cossich ACC, Fonseca ME, Ferreira VJAA. Estudo retrospectivo sobre a audição de trabalhadores expostos ao ruído. Rev CEFAC. 2002; 4(1):145-8.

15. Fernandes M, Morata TC. Estudo dos efeitos auditivos e extra-auditivos da exposição ocupacional a ruído e vibração. Rev Bras Otorrinolaringol. 2002; 68(5):705-13.

16. Dias A, Cordeiro R, Gonçalves CGO. Exposição ocupacional ao ruído e acidentes do trabalho. Cad Saúde Pública. 2006; 22(10):2125-30.

17. Cordeiro R, Clemente APG, Diniz CS, Dias A. Exposição ao ruído ocupacional como fator de risco para acidentes do trabalho. Rev Saúde Pública. 2005; 39(3):461-6.

18. Ferreira P, Peloggia CCS, Pirinoto H F, Bernardi APA. Influência da conservação do protetor auricular na prevenção da perda auditiva induzida por níveis de pressão sonora elevados. Rev CEFAC. 2003; 5(1):353-9.

19. Gonçalves CGO, Couto CM, Carraro JM, Leonelli BS. Avaliação da colocação de protetores auriculares em grupos com e sem treinamento. Rev CEFAC. 2009; 11(2):345-52. dx.doi.org/10.1590/ S1516-18462009000200021

20. Jacob LCB, Aguiar FP, Tomiasi AA, Tschoeke SN, Bitencourt RF. Monitoramento auditivo na ototoxidade. Rev Bras Otorrinolaringol. 2006; 72(6):836-44.

21. Botelho CT, Paz APML, Gonçalves AM, Frota S. Estudo comparativo de exames audiométricos de metalúrgicos expostos a ruído e ruído associado a produtos químicos. Rev Bras Otorrinolaringol. 2009; 75(1):51-7.

22. Steinmetz LG, Zeigelboim BS, Lacerda $A B$, Morata TC, Marques JM. Características do zumbido em trabalhadores expostos a ruído. Rev Bras Otorrinolaringol. 2009; 75(1):7-14.

23. Blandino PC, Garcia AP. Estudo das queixas auditivas e extra-auditivas em trabalhadores do serviço social do comércio. Acta ORL. 2006; 24(4):239-44.

24. Gonçalves CGO, Mota PHM, Marques JM. Ruído e idade: análise da influência na audição em indivíduos com 50 - 70 anos. Pró-Fono. 2009; 21(1):57-62.

25. Veras RP, Matos LC. Audiologia do envelhecimento: revisão da literatura e perspectivas atuais. Rev Bras Otorrinolaringol. 2007; 73(1): 128-34.

RECEBIDO EM: 27/04/2009

ACEITO EM: 21/10/2009

Endereço para correspondência:

Luciana Dias Bernardo

Av. Agostinho Bianchetti, 124

Alfredo Vasconcelos - MG

CEP: 36272-000

E-mails: ludiasb@gmail.com lucdbernardo@ hotmail.com 